\title{
Modification of Cycloserine Cefoxitin Fructose Agar to Suppress Growth of Yeasts from Stool Specimens
}

\author{
Kyungwon Lee, Dongeun Yong, Jonghwa Yum, \\ Young Suk Sohn and Yunsop Chong*
}

Department of Clinical Pathology and Research Institute of

Bacterial Resistance, Yonsei University College of Medicine, C.P.O. Box 8044, Seoul, Korea

(Received 15 June 1999; accepted in revised form 3 July 2000)

Key Words: C. difficile, CCFA, amphotericin B-supplemented CCFA, yeast suppression
Cycloserine cefoxitin fructose agar is a widely used selective isolation medium for Clostridium difficile from stool specimens. Yeasts often colonize in the intestine of $C$. difficile disease patients and, if colonized heavily, pure culture of $C$. difficile can be delayed. The aim of this study was to modify cycloserine cefoxitin fructose agar to suppress the growth of yeasts. Antimicrobial activities of three commonly available antifungal agents were tested against recent clinical isolates of Candida species. Amphotericin B was most active in inhibiting all isolates by $\leq 0.5 \mathrm{mg} / \mathrm{L}$ concentration. Cycloserine cefoxitin fructose agar was modified by adding $2 \mathrm{mg} / \mathrm{L}$ of amphotericin B. Serial ten-fold dilution of stool specimens from 126 suspected C. difficile-associated diarrhea patients were cultured both on cycloserine cefoxitin fructose agar plates and modified agar plates. Yeasts grew from 60 specimens on cycloserine cefoxitin fructose agar, but none grew on the modified medium. Growth of $C$. difficile was detected from 37 and 39 of 126 specimens on cycloserine cefoxitin fructose agar and modified medium, respectively. The number of $C$. difficile colonies was similar on both media. In conclusion, $2 \mathrm{mg} / \mathrm{L}$ of amphotericin $\mathrm{B}$ supplementation to cycloserine cefoxitin fructose agar can facilitate the isolation of $C$. difficile from stool specimens which are densely colonized with yeasts.

(C) 2000 Academic Press

\section{Introduction}

Clostridium difficile causes $10-20 \%$ of all cases of antibiotic-associated diarrhea and nearly all cases of antibiotic associated pseudomembranous colitis [1].

*Address correspondence to: Department of Clinical Pathology and Research Institute of Bacterial Resistance, Yonsei University College of Medicine, C.P.O. Box 8044, Seoul, Korea.

E-mail: whonetkor@yumc.yonsei.ac.kr
C. difficile is a significant nosocomial pathogen. One study estimated the cost of treating a single C. difficileassociated diarrhea (CDAD) infection to be in excess of $£ 4000$ [2].

Culture of $C$. difficile from stool specimens is the most useful tool for the epidemiological studies [3,4]. Also, as the toxin is not always detected in stools of some CDAD patients, culture and subsequent toxin test is useful for the diagnosis of additional patients [5]. In a Canadian survey, $32.1 \%$ of hospitals used C. difficile culture either as the only test or together 
with other tests [6]. Quantitative culture [7] may be useful to differentiate a normal carrier with small number of $C$. difficile in their stool.

Various media and their modifications are used for the isolation of $C$. difficile from stool specimens. Cycloserine cefoxitin fructose agar (CCFA) has been widely used because it efficiently suppressed most of the bacterial species present in stool specimens [8]. However, with the increase of antimicrobial-resistant bacteria, we have been experiencing frequent growth of intestinal bacterial flora on CCFA plates. The growth of intestinal bacteria did not hinder the isolation and identification of $C$. difficile because of their distinct morphology and smaller size. Most of the stool specimens for $C$. difficile cultures are from inpatients who are treated with broad-spectrum antimicrobial agents. Therefore, we have been increasingly experiencing stool specimens which contain a large number of yeasts that make it difficult to determine colony-forming units of $C$. difficile. Also subculture is often required to obtain pure cultures. For the selective isolation of certain fastidious bacteria, various antifungal agents are also incorporated to suppress yeasts. However, CCFA does not contain any antifungal agents.

In this study, we compared antifungal activities of amphotericin B, fluconazole and 5-fluorocytocine against recent clinical isolates of Candida spp. Based on the test, we selected amphotericin B as a candidate to supplement CCFA. We evaluated the effects of adding amphotericin $\mathrm{B}$ to CCFA for the isolation and enumeration of $C$. difficile and the suppression of yeast from stool culture.

\section{Materials and Methods}

Candida spp. were isolated from various clinical specimens. Minimum inhibitory concentrations (MICs) of amphotericin B (Bristol-Meyers Squibb Co., Princeton, NJ, U.S.A.), fluconazole (Pfizer Inc., New York, NY, U.S.A.), and 5-fluorocytosine (Sigma Chemical, St. Louis, MO, U.S.A.) were determined by the broth microdilution test [9] using RPMI medium 1640 (Gibco Laboratories, Grand Island, NY, U.S.A.)

CCFA plates were prepared by adding $5 \%$ of egg yolk emulsion and Clostridium-selective supplement (Oxoid, Basingstoke, U.K.) to autoclave-sterilized Clostridium difficile agar base (Oxoid). Final concentrations of cycloserine and cefoxitin were $500 \mathrm{mg} / \mathrm{L}$ and $16 \mathrm{mg} / \mathrm{L}$, respectively. Amphotericin B-supplemented CCFA (CCFA-AMB) were prepared by adding amphotericin $B$ to CCFA to a final concentration of $2 \mathrm{mg} / \mathrm{L}$.

Soft or fluid stool specimens were collected from suspected CDAD patients in a tertiary-care hospital.
Serial ten-fold dilutions of stool specimens were prepared according to the method of Summanen et al. [7] with modification; thioglycollate medium without dextrose or indicator (Difco, Detroit, MI, U.S.A.) was used instead of $0.05 \%$ yeast extract solution. Then, $0.1 \mathrm{~mL}$ amounts of $10^{-2}, 10^{-3}$ and $10^{-4}$ dilutions of specimens were spread onto both CCFA and CCFA-AMB plates. The plates were incubated in an anaerobic chamber (Forma Scientific Inc., Marietta, OH, U.S.A.) for $48 \mathrm{~h}$ and the number of colonies of suspected $C$. difficile and yeasts were counted. Suspected $C$. difficile isolates were identified by ATB rapid ID 32A (bioMerieux sa, Marcy-l'Etoile, France). Gram-stained smears of suspected yeast colonies were observed microscopically, but species were not identified.

\section{Results and Discussion}

Laboratory diagnosis is required for the proper management of CDAD patients. For the laboratory diagnosis of CDAD, cytotoxin (toxin B) detection is still considered the gold standard, but as many as $15 \%$ to $38 \%$ of patients with confirmed CDAD were not detected by direct stool cytotoxin neutralization assay [10]. Stool cultures alone are not sufficient for the diagnosis of CDAD, as some strains are non-toxigenic and hence non-pathogenic. However, isolation of a toxigenic strain can detect additional patients and it is also necessary for epidemiological study.

In this study, the growth of all C. parapsilosis isolates was inhibited by $2 \mathrm{mg} / \mathrm{L}$ or less of fluconazole or 5 -fluorocytosine, but some isolates of other species were inhibited only by a much higher concentration. Amphotericin B $0.5 \mathrm{mg} / \mathrm{L}$ or less inhibited all of the Candida strains tested (Table 1). Amphotericin B was selected for the modification of CCFA because it not only has strong antifungal activity, but it is also easily available in hospitals.

Among the 126 stool specimens cultured, 47.7\% yielded yeasts on CCFA. The number of yeast colonies developed on CCFA ranged from $10^{3}$ to $\geq 10^{6} \mathrm{CFU} /$ $\mathrm{mL}$, and the count was $\geq 10^{6}$ in $33.3 \%$ of specimens.

Table 1. Antifungal activities of amphotericin B, fluconazole and 5-fluorocytosine against clinical isolates of Candida spp.

\begin{tabular}{lccc}
\hline Candida spp. & \multicolumn{3}{c}{ MIC ranges (mg/L) of: } \\
\cline { 2 - 4 } (No. tested) & Amphotericin B & Fluconazole & 5-fluorocytosine \\
\hline C. albicans (60) & $0.06-0.25$ & $0.12->64$ & $\leq 0.12->64$ \\
C. tropicalis (14) & $0.12-0.25$ & $2->64$ & $\leq 0.12-0.5$ \\
C parapsilosis (10) & $0.06-0.12$ & $0.5-2$ & $\leq 0.12$ \\
C. lusitaniae (4) & $0.12-0.25$ & $2->64$ & $\leq 0.12-0.25$ \\
C. famata (2) & $0.12-0.5$ & $1->64$ & $\leq 0.12$ \\
C. krusei (2) & $0.25-0.5$ & $\geq 64$ & $1-8$ \\
\hline
\end{tabular}


Table 2. Comparison of yeast growth on CCFA and CCFA-AMB from stool specimens

\begin{tabular}{lcc}
\hline Yeast growth $(\mathrm{CFU} / \mathrm{mL})$ & \multicolumn{2}{c}{$\begin{array}{c}\text { No. (\%) of specimens with yeast } \\
\text { growth on: }\end{array}$} \\
\cline { 2 - 3 } & CCFA & CCFA-AMB \\
\hline$<10^{2 \mathrm{~b}}$ & $66(52.3)$ & $126(100)$ \\
$10^{3}$ & $1(0.7)$ & $0(0)$ \\
$10^{4}$ & $7(5.5)$ & $0(0)$ \\
$10^{5}$ & $10(7.9)$ & $0(0)$ \\
$\geq 10^{6}$ & $42(33.3)$ & $0(0)$ \\
Total & $126(100)$ & $126(100)$ \\
\hline
\end{tabular}

${ }^{\mathrm{a}}$ Amphotericin B-supplemented CCFA.

${ }^{b}$ No growth.

CCFA-AMB completely inhibited the growth of yeasts (Table 2).

A small number of $C$. difficile in a stool should be less significant than a large number organisms. To determine the number of $C$. difficile in stool specimens, the quantitative culture method was used [7]. The lowest detection limit of $C$. difficile in this study was $10^{2} \mathrm{CFU} / \mathrm{mL}$. C. difficile was isolated in 37 of 126 stool specimens ioculated on CCFA, while the number was 39 on CCFA-AMB (Table 3). Among the C. difficilepositive specimens, the number of $C$. difficile colonies was similar and mostly $\geq 10^{6} \mathrm{CFU} / \mathrm{mL}$ on both media. It should be natural to see that the addition of an antifungal agent did not adversely affect the growth of $C$. difficile (Table 3). From two samples, growth of C. difficile was detected on CCFA-AMB, but not on CCFA: one sample was with $10^{6} \mathrm{CFU} / \mathrm{mL}$ of yeasts and another sample was with heavy growth of bacteria. From another sample with $10^{5} \mathrm{CFU} / \mathrm{mL}$ of yeasts, ten-fold more $\mathrm{CFU} / \mathrm{mL}$ of $C$. difficile were detected on CCFA-AMB. These differences may be due to the presence of yeasts or bacteria.

Aerobic and anaerobic bacteria grew from some specimens on both CCFA and CCFA-AMB. The species were mostly Enterococcus, Klebsiella pneumoniae, Enterobacter spp., Serratia marcescens and Pseudomonas aeruginosa, which are the species most often resistant to antimicrobial agents. Further improvement of selectivity of CCFA-AMB may facilitate the pure culture of $C$. difficile. However, we considered that this is not necessary because colonies of these organisms can be differentiated from that of $C$. difficile by their distinct colony size and morphology.

In conclusion, amphotericin $\mathrm{B}$ can be added to a final concentration of $2 \mathrm{mg} / \mathrm{L}$ to CCFA to inhibit the growth of yeasts, thereby to facilitate the isolation of a
Table 3. Comparison of growth of $C$. difficile on CCFA and CCFAAMB from stool specimens

\begin{tabular}{|c|c|c|c|c|c|}
\hline \multirow[t]{2}{*}{ Growth $(\mathrm{CFU} / \mathrm{mL})$ on } & \multicolumn{5}{|c|}{$\begin{array}{l}\text { No. of specimens with growth } \\
(\mathrm{CFU} / \mathrm{mL}) \text { on CCFA-AMB }\end{array}$} \\
\hline & $10^{2}$ & $10^{3}$ & $10^{4}$ & $10^{5}$ & $\geq 10^{6}$ \\
\hline $\begin{array}{l}<10^{2} \mathrm{~b}(2) \\
10^{2}(1) \\
10^{3}(2) \\
10^{4}(2) \\
10^{5}(3) \\
\geq 10^{6}(29)\end{array}$ & $\begin{array}{l}1^{\mathrm{c}} \\
1\end{array}$ & 1 & $\begin{array}{l}1^{\mathrm{c}} \\
2\end{array}$ & 3 & 29 \\
\hline Total (39) & 2 & 2 & 3 & 3 & 29 \\
\hline
\end{tabular}

pure culture and the determination of colony forming units of $C$. difficile from stool specimens.

\section{References}

1. Bartlett J.G. (1994) Clostridium difficile: history of its role as an enteric pathogen and the current state of knowledge about the organism. Clin Infect Dis 18: S265-272

2. Wilcox M.H., Cunniffe J.G., Trundle C. and Redpath C. (1999) Financial burden of hospital acquired Clostridium difficile infection. J Hosp Infect 34: 23-30

3. George W.L. (1989). Antimicrobial agent-associated diarrhea and colitis. In Finegold S.M. and George W.L. (eds) Anaerobic Infections in Humans, pp 661-678. Academic Press, San Diego, CA

4. Merz C.S., Kramert C., Forman M., Gluck L., Mills K., Senft K., Steiman I., Wallace N. and Charache P. (1994) Comparison of four commercially available rapid enzyme immunoassays with cytotoxin assay for detection of Clostridium difficile toxin(s) from stool specimens. J Clin Microbiol 32: 1142-1147

5. Bond F.B., Payne G., Borriello S.P. and Humphreys H. (1995) Usefulness of culture in the diagnosis of Clostridium difficile infection. Eur J Clin Microbiol Infect Dis 14: 223-226

6. Alfa M.J. and Beda G. (1998) Survey of incidence of Clostridium difficile infection in Canadian hospitals and diagnostic approaches. J Clin Microbiol 36: 2076-2080

7. Summanen P., Baron E.J., Citron D.M., Strong C., Wexler H.M. and Finegold S.M. (1993) Wadsworth Anaerobic Bacteriology Manual. 5th Edn p 95-101, Star Publishing Co., Belmont, CA

8. George W.L., Sutter V.L., Citron D. and Finegold S.M. (1979) Selective and differential medium for isolation of Clostridium difficile. J Clin Microbiol 9: 214-219

9. National Committee for Clinical Laboratory Standards (1995) Reference method for broth dilution antifungal susceptibility testing of yeasts; tentative standard. M27-T. National Committee for Clinical Laboratory Standards. Wayne, PA, U.S.A.

10. Staneck J.L., Weckbach L.S., Allen D.S., Siders J.A., Gilligan P.H., Coppitt G., Kraft J.A. and Willis D.H. (1996) Multicenter evaluation of four methods for Clostridium difficile detection: ImmunoCard C. difficile, cytotoxin assay, culture, and latex agglutination. J Clin Microbiol 34: 2718-2721 